\title{
Low-Temperature Adsorption and Diffusion of Methanol in ZIF-8 Nanoparticle Films
}

Amber M. Mosier, ${ }^{\dagger}$ Hanna L. W. Larson, ${ }^{\dagger}$ Elizabeth R. Webster, ${ }^{\dagger}$ Mia Ivos, ${ }^{\dagger}$ Fangyuan Tian, ${ }^{\star}$ Lauren Benz ${ }^{\dagger, *}$

†Department of Chemistry \& Biochemistry, University of San Diego, San Diego, California, 92110, United States

${ }^{ \pm}$Department of Chemistry \& Biochemistry, California State University, Long Beach, California, 90840, United States

\section{Supporting Information}


Temperature Programmed Desorption (TPD) Studies of Methanol on a $\mathrm{TiO}_{2}(110)$ Surface

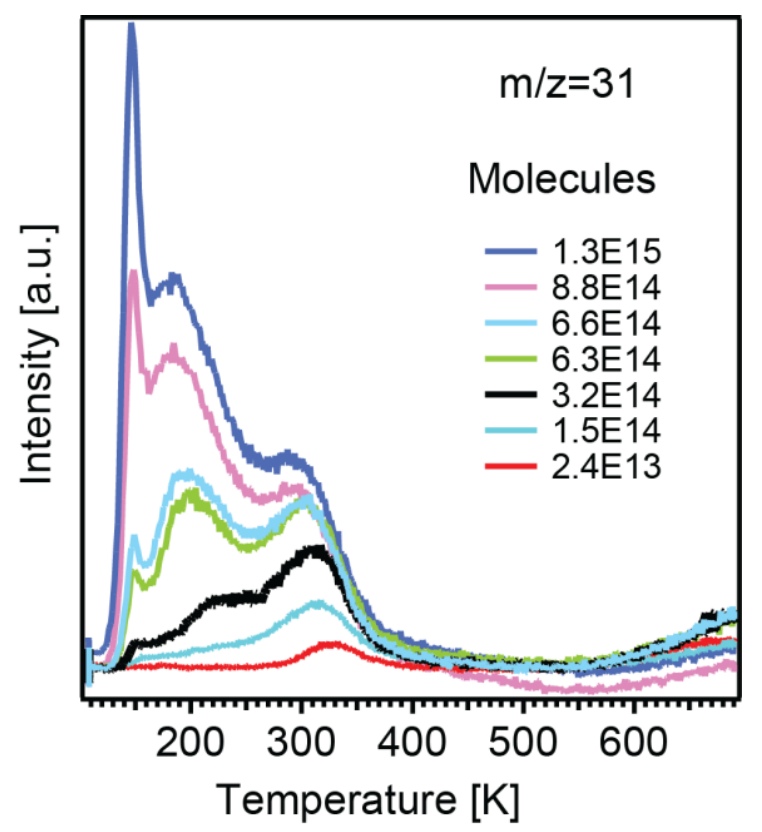

Figure S1. TPD spectra of methanol desorption from a $\mathrm{TiO}_{2}(110)$ surface following adsorption at $90 \mathrm{~K}$. The black spectrum indicates a monolayer of methanol formed on a $\mathrm{TiO}_{2}(110)$ surface. Note that prior to examination the $\mathrm{TiO}_{2}(110)$ surface was prepared with several cycles of $\mathrm{Ar}+$ bombardment and annealing following previously published preparation methods until a carbon signal was no longer visible by XPS. ${ }^{1}$

\section{Control Studies: Methanol Adsorption on a Au Surface}

To examine the influence of ZIF-8 nanoparticle surface coverage on methanol adsorption, we performed control experiments on a $\mathrm{Au}$ substrate representative of the underlying Au surface present during the preparation of our ZIF-8 films. Our Au surface is a Au-coated silicon wafer with a $50 \pm 5 \mathrm{~nm} \mathrm{Au}$ film on a $500 \pm 30 \mu \mathrm{m}$ p-type $\mathrm{Si}(111)$ substrate. 


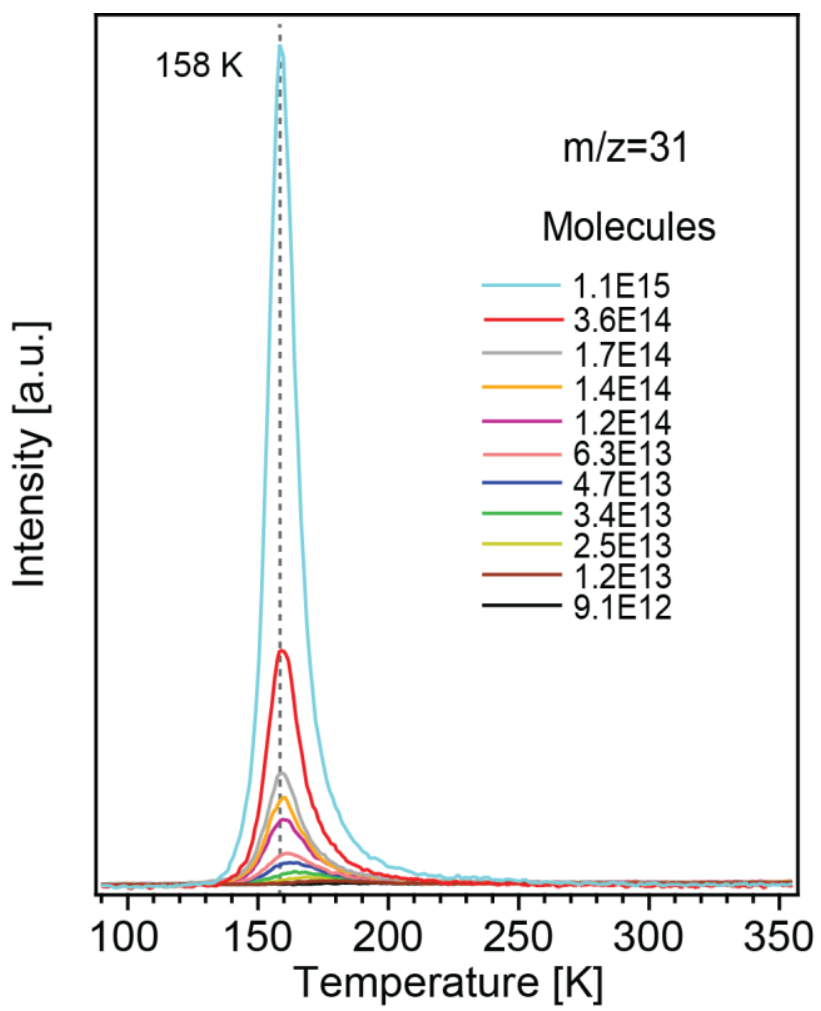

Figure S2. TPD spectra of methanol desorption from a Au surface following exposure to methanol at $90 \mathrm{~K}$.

The desorption spectra of methanol are presented in Figure S2, with sub-monolayer desorption starting at $\sim 180 \mathrm{~K}$ and shifting to $158 \mathrm{~K}$ with increasing exposure, the highest trace at $1.1 \times 10^{15}$ molecules being just above the estimated monolayer coverage on $\mathrm{Au}$ of $7.16 \times 10^{14}$ molecules $/ \mathrm{cm}^{2}{ }^{2}$ The temperature of the multilayer is approximately $20 \mathrm{~K}$ greater than the previously reported desorption temperature in the literature for multilayers on a pristine in-situ cleaned $\mathrm{Au}(111)$ surface, ${ }^{3}$ however in this case we use an as-installed Au-coated $\mathrm{Si}$ wafer (which is our true control) in which the thermocouple was affixed mainly to the Si. 


\section{Methanol Adsorption on a 2-cycle ZIF-8 Surface}

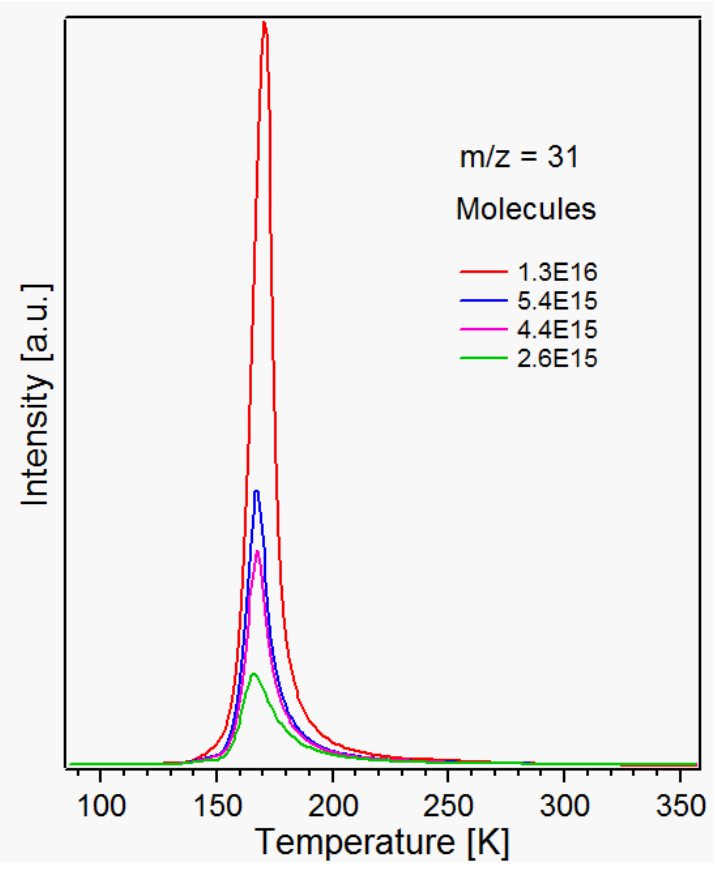

Figure S3. Methanol desorption from a 2-cycle ZIF-8 sample at larger exposures (exposure temperature $90 \mathrm{~K}$ ).

Figure S3 is a continuation of Figure 1 in the MS of greater exposures. The peak continues to grow and shifts slightly to higher temperature, consistent with zeroth order behavior. No new features were observed.

\section{Butanol TPD and XPS Studies at $90 \mathrm{~K}$}

Figure S4 shows a series of TPD spectra following exposure to butanol at $90 \mathrm{~K}$. Since butanol has a longer carbon chain and therefore a greater molecular surface area, it exhibits stronger interactions with itself and ZIF-8. The desorption temperature in the limit of low coverage is $213 \mathrm{~K}$, and with increased exposure the desorption peak 
temperature decreases to $201 \mathrm{~K}$. These temperatures are approximately $40 \mathrm{~K}$ greater than that of methanol, but the overall peak shape (and the fact that there is only one peak) is similar to the case of methanol desorption.

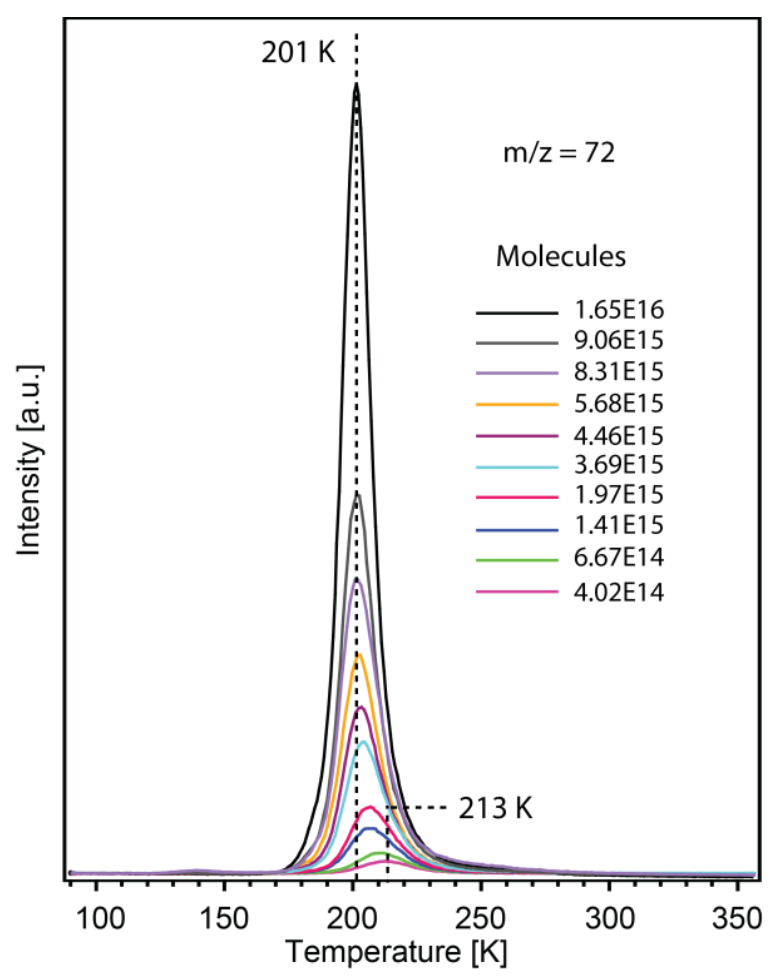

Figure S4. TPD spectra of butanol desorption following exposure of a 2-cycle ZIF-8 film to butanol at $90 \mathrm{~K}$.

Figure S5 shows the XPS O 1s and C 1s spectra following the exposure of a 2cycle ZIF-8 nanoparticle film to $1.6 \times 10^{15}$ butanol molecules. Large peaks become visible in the $\mathrm{O} 1 \mathrm{~s}$ and $\mathrm{C} 1 \mathrm{~s}$ regions corresponding to butanol. Appearance of these strong peaks similar to what was observed in the case of methanol suggests that butanol, like methanol, is partitioned between surface and pore sites at $90 \mathrm{~K}$. For reference these peaks appear at a coverage which corresponds to roughly $2.75 \mathrm{ML}$ of butanol on a flat $\mathrm{TiO}_{2}(110)$ surface $^{4}$ (recall from the MS that for water which accumulates nearly 
exclusively at the surface, only 0.4 ML was required before an XPS signal was clearly visible on a ZIF-8 film, so this suggests some pore penetration in addition to surface accumulation of butanol). Note that we used the TPD calibration for methanol on the molecular count here assuming that the response of the mass spectrometer to $\mathrm{C} 1$ and $\mathrm{C} 4$ alcohols should be comparable.
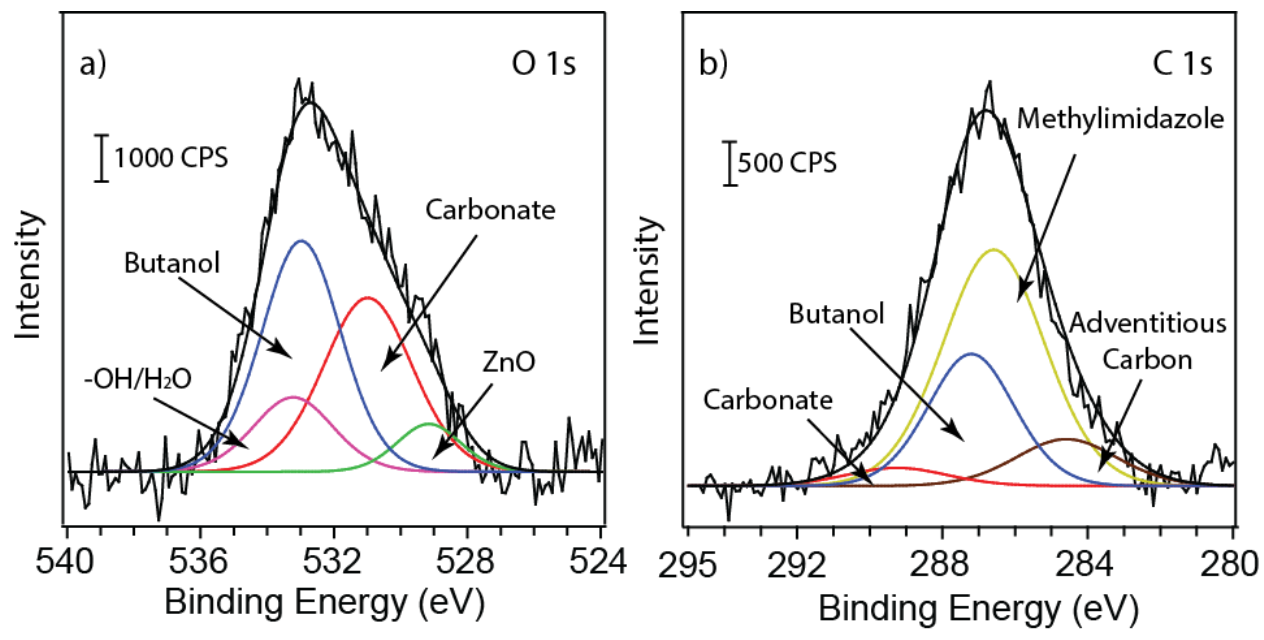

Figure S5. XPS spectra of butanol following exposure of a 2-cycle ZIF-8 film to butanol at $90 \mathrm{~K}$.

\section{REFERENCES}

(1) Benz, L.; Park, A.; Corey, J. R.; Mezher, M. P.; Park, V. C. Interaction of Petroleum-Rlevant Organosulfur Compounds with $\mathrm{TiO}_{2}(110)$. Langmuir 2012, 28, 10209-10216.

(2) Green, S. D.; Bolina, A. S.; Chen, R.; Collings, M. P.; Brown, W. A.; McCoustra, M. R. S. Applying Laboratory Thermal Desorption Data in an Interstellar Context: Sublimation of Methanol Thin Films(Star). Monthly Notices of the Royal Astronomical Society 2009, 398, 357367.

(3) Gong, J.; Flaherty, D. W.; Ojifinni, R. A.; White, J. M.; Mullins, C. B. Surface Chemistry of Methanol on Clean and Atomic Oxygen Pre-Covered Au(111). J. Phys. Chem. C 2008, 112, 5501-5509.

(4) Li, Z. J.; Smith, R. S.; Kay, B. D.; Dohnalek, Z. Determination of Absolute Coverages for Small Aliphatic Alcohols on TiO2(110). J. Phys. Chem. C 2011, 115, 22534-22539. 
